Kragujevac Journal of Mathematics

Volume 44(3) (2020), Pages 475-485.

\title{
SOME ESTIMATES FOR HOLOMORPHIC FUNCTIONS AT THE BOUNDARY OF THE UNIT DISC
}

\author{
B. N. ORNEK ${ }^{1}$
}

\begin{abstract}
In this paper, for holomorphic function $f(z)=z+c_{2} z^{2}+c_{3} z^{3}+\cdots$ belong to the class of $\mathcal{N}(\lambda)$, it has been estimated from below the modulus of the angular derivative of the function $\frac{z f^{\prime}(z)}{f(z)}$ on the boundary point of the unit disc.
\end{abstract}

\section{INTRODUCTION}

Let $f$ be a holomorphic function in the unit disc $E=\{z:|z|<1\}, f(0)=0$ and $|f(z)|<1$ for $|z|<1$. In accordance with the classical Schwarz lemma, for any point $z$ in the disc $E$, we have $|f(z)| \leq|z|$ and $\left|f^{\prime}(0)\right| \leq 1$. Equality in these inequalities (in the first one, for $z \neq 0$ ) occurs only if $f(z)=z e^{i \theta}$, where $\theta$ is a real number ([8], p. 329). For historical background about the Schwarz lemma and its applications on the boundary of the unit disc, we refer to (see $[2,7]$ ).

The basic tool in proving our results is the following lemma due to Jack.

Lemma 1.1 (Jack's lemma). Let $f(z)$ be holomorphic function in the unit disc $E$ with $f(0)=0$. Then if $|f(z)|$ attains its maximum value on the circle $|z|=r$ at a point $z_{0} \in E$, then there exists a real number $k \geq 1$ such that

$$
\frac{z_{0} f^{\prime}\left(z_{0}\right)}{f\left(z_{0}\right)}=k
$$

Let $\mathcal{A}$ denote the class of functions

$$
f(z)=z+c_{2} z^{2}+c_{3} z^{3}+\cdots
$$

Key words and phrases. Schwarz lemma, holomorphic function, angular limit. 2010 Mathematics Subject Classification. Primary: 30C80. Secondary: 32A10.

DOI 10.46793/KgJMat2003.475O

Received: August 02, 2017.

Accepted: June 06, 2018. 
that are holomorphic in the unit disc $E$. Also, $\mathcal{N}(\lambda)$ be the subclass of $\mathcal{A}$ consisting of all functions $f(z)$ which satisfy

$$
\left|\frac{z f^{\prime}(z)}{f(z)}\right|^{\alpha}\left|z\left(\frac{z f^{\prime}(z)}{f(z)}\right)^{\prime}\right|^{\beta}<\left(\frac{1}{2} \lambda\right)^{\beta},
$$

for some real $\alpha \geq 0, \beta>0$ and $\lambda=\frac{\beta}{\beta+\alpha}$.

Let $f(z) \in \mathcal{N}(\lambda)$ and define $\phi(z)$ in $E$ by

$$
\phi(z)=\frac{(h(z))^{\frac{1}{\lambda}}-1}{(h(z))^{\frac{1}{\lambda}}+1},
$$

where $h(z)=\frac{z f^{\prime}(z)}{f(z)}$.

Obviously, $\phi(z)$ is holomorphic function in the unit disc $E$ and $\phi(0)=0$. We want to prove $|\phi(z)|<1$ for $|z|<1$. Differentiating (1.2) and simplifiying, we obtain

$$
\left(\frac{z f^{\prime}(z)}{f(z)}\right)^{\prime}=\frac{2 \lambda \phi^{\prime}(z)}{(1-\phi(z))^{2}}\left(\frac{1+\phi(z)}{1-\phi(z)}\right)^{\lambda-1}
$$

and, so

$$
\begin{aligned}
\left|\frac{z f^{\prime}(z)}{f(z)}\right|^{\alpha}\left|z\left(\frac{z f^{\prime}(z)}{f(z)}\right)^{\prime}\right|^{\beta} & =\left|\frac{1+\phi(z)}{1-\phi(z)}\right|^{\alpha \beta+\beta(\lambda-1)}\left|\frac{2 \lambda z \phi^{\prime}(z)}{(1-\phi(z))^{2}}\right|^{\beta} \\
& =\left|\frac{2 \lambda z \phi^{\prime}(z)}{(1-\phi(z))^{2}}\right|^{\beta}<\left(\frac{\lambda}{2}\right)^{\beta} .
\end{aligned}
$$

If there exists a point $z_{0} \in E$ such that

$$
\max _{|z| \leq\left|z_{0}\right|}|\phi(z)|=\left|\phi\left(z_{0}\right)\right|=1
$$

then Jack's lemma gives us that $\phi\left(z_{0}\right)=e^{i \theta}$ and $z_{0} \phi^{\prime}\left(z_{0}\right)=k \phi\left(z_{0}\right), k \geq 1$.

Thus we have

$$
\begin{aligned}
\left|\frac{z_{0} f^{\prime}\left(z_{0}\right)}{f\left(z_{0}\right)}\right|^{\alpha}\left|z_{0}\left(\frac{z_{0} f^{\prime}\left(z_{0}\right)}{f\left(z_{0}\right)}\right)^{\prime}\right|^{\beta} & =\left|\frac{2 \lambda z_{0} \phi^{\prime}\left(z_{0}\right)}{\left(1-\phi\left(z_{0}\right)\right)^{2}}\right|^{\beta}=\left|\frac{2 \lambda k e^{i \theta}}{\left(1-e^{i \theta}\right)^{2}}\right|^{\beta} \\
& =\frac{(2 \lambda k)^{\beta}}{\left|1-e^{i \theta}\right|^{2 \beta}} \geq \frac{(2 \lambda)^{\beta}}{2^{2 \beta}}=\left(\frac{\lambda}{2}\right)^{\beta} .
\end{aligned}
$$

This contradict (1.1). So, there is no point $z_{0} \in E$ such that $\phi\left(z_{0}\right)=1$. This means that $|\phi(z)|<1$ for $|z|<1$. Thus, from the Schwarz lemma, we obtain

$$
\left|c_{2}\right| \leq \frac{2 \beta}{\beta+\alpha} .
$$

Moreover, the equality $\left|c_{2}\right|=\frac{2 \beta}{\beta+\alpha}$ occurs for the function

$$
f(z)=e^{\int^{z} \frac{1}{t}\left(\frac{1+t}{1-t}\right)^{\lambda} d t}
$$


That proves the following lemma.

Lemma 1.2. If $f(z) \in \mathcal{N}(\lambda)$, then we have

$$
\left|c_{2}\right| \leq \frac{2 \beta}{\beta+\alpha} .
$$

The equality in (1.3) occurs for the function

$$
f(z)=e^{\int^{z} \frac{1}{t}\left(\frac{1+t}{1-t}\right)^{\lambda} d t} .
$$

The following boundary version of the Schwarz lemma was proved in 1938 by Unkelbach in [21] and then rediscovered and partially improved by Osserman in [17].

Lemma 1.3. Let $f(z)$ be a holomorphic function self-mapping of $E=\{z:|z|<1\}$, that is $|f(z)|<1$ for all $z \in E$. Assume that there is a $b \in \partial E$ so that $f$ extend continuously to $b,|f(b)|=1$ and $f^{\prime}(b)$ exists. Then

$$
\left|f^{\prime}(b)\right| \geq \frac{2}{1+\left|f^{\prime}(0)\right|} .
$$

The equality in (1.4) holds if and only if $f$ is of the form

$$
f(z)=-z \frac{a-z}{1-a z}, \quad \text { for all } z \in E,
$$

for some constant $a \in(-1,0]$.

Corollary 1.1. Under the hypotheses lemma, we have

$$
\left|f^{\prime}(b)\right| \geq 1
$$

with equality only if $f$ is of the form

$$
f(z)=z e^{i \theta}
$$

where $\theta$ is a real number.

The following Lemma 1.4 and Corollary 1.2, known as the Julia-Wolff lemma, is needed in the sequel [15].

Lemma 1.4 (Julia-Wolff lemma). Let $f$ be a holomorphic function in $E, f(0)=0$ and $f(E) \subset E$. If, in addition, the function $f$ has an angular limit $f(b)$ at $b \in \partial E$, $|f(b)|=1$, then the angular derivative $f^{\prime}(b)$ exists and $1 \leq\left|f^{\prime}(b)\right| \leq \infty$.

Corollary 1.2. The holomorphic function $f$ has a finite angular derivative $f^{\prime}(b)$ if and only if $f^{\prime}$ has the finite angular limit $f^{\prime}(b)$ at $b \in \partial E$.

Inequality (1.4) and its generalizations have important applications in geometric theory of functions (see, e.g., $[8,18]$ ). Therefore, the interest to such type results is not vanished recently (see, e.g., $[1,2,5-7,15-17,19,20]$ and references therein). 
Vladimir N. Dubinin has continued this line and has made a refinement on the boundary Schwar lemma under the assumption that $f(z)=c_{p} z^{p}+c_{p+1} z^{p+1}+\cdots$, with a zero set $\left\{z_{k}\right\}$ (see [5]).

S. G. Krantz and D. M. Burns [3] and D. Chelst [4] studied the uniqueness part of the Schwarz lemma. According to M. Mateljević's studies, some other types of results which are related to the subject can be found in ([13,14] and [12]). In addition, [11] was posed on ResearchGate where is discussed concerning results in more general aspects.

Also, M. Jeong [10] showed some inequalities at a boundary point for different form of holomorphic functions and found the condition for equality and in [9] a holomorphic self map defined on the closed unit disc with fixed points only on the boundary of the unit disc.

\section{MAin Results}

In this section, for holomorphic function $f(z)=z+c_{2} z^{2}+c_{3} z^{3}+\cdots$ belong to the class of $\mathcal{N}(\lambda)$, it has been estimated from below the modulus of the angular derivative of the function $\frac{z f^{\prime}(z)}{f(z)}$ on the boundary point of the unit disc.

Theorem 2.1. Let $f(z) \in \mathcal{N}(\lambda)$. Assume that, for some $b \in \partial E$, $f$ has angular limit $f(b)$ at $b$ and $\frac{b f^{\prime}(b)}{f(b)}=i^{\lambda}$. Then we have the inequality

$$
\left|\left(\frac{z f^{\prime}(z)}{f(z)}\right)_{z=b}^{\prime}\right| \geq \frac{\beta}{\beta+\alpha} .
$$

The equality in (2.1) occurs for the function

$$
f(z)=e^{\int^{z} \frac{1}{t}\left(\frac{1+t}{1-t}\right)^{\lambda} d t}
$$

where $\lambda=\frac{\beta}{\beta+\alpha}$.

Proof. Consider the function

$$
\phi(z)=\frac{(h(z))^{\frac{1}{\lambda}}-1}{(h(z))^{\frac{1}{\lambda}}+1}
$$

where $h(z)=\frac{z f^{\prime}(z)}{f(z)}$ and $\lambda=\frac{\beta}{\beta+\alpha} . \phi(z)$ is a holomorphic function in the unit disc $E$ and $\phi(0)=0$. From the Jack's lemma and since $f(z) \in \mathcal{N}(\lambda)$, we obtain $|\phi(z)|<1$ for $|z|<1$. Also, we have $|\phi(b)|=1$ for $b \in \partial E$.

From (1.5), we obtain

$$
1 \leq\left|\phi^{\prime}(b)\right|=\frac{2}{\lambda}\left|\frac{(h(b))^{\frac{1}{\lambda}-1} h^{\prime}(b)}{\left(1+(h(b))^{\frac{1}{\lambda}}\right)^{2}}\right|=\frac{2}{\lambda}\left|\frac{\left(i^{\lambda}\right)^{\frac{1}{\lambda}-1} h^{\prime}(b)}{\left(1+\left(i^{\lambda}\right)^{\frac{1}{\lambda}}\right)^{2}}\right|=\frac{2}{\lambda}\left|\frac{\left(i^{\lambda}\right)^{\frac{1}{\lambda}-1} h^{\prime}(b)}{\left(1+\left(i^{\lambda}\right)^{\frac{1}{\lambda}}\right)^{2}}\right|
$$


and

$$
1 \leq \frac{2}{\lambda} \frac{\left|h^{\prime}(b)\right|}{|1+i|^{2}}=\frac{\left|h^{\prime}(b)\right|}{\lambda} .
$$

So, we take the inequality (2.1).

Now, we shall show that the inequality (2.1) is sharp. Let

$$
f(z)=e^{\int_{0}^{z} \frac{1}{t}\left(\frac{1+t}{1-t}\right)^{\lambda} d t}
$$

Then, we have

$$
\begin{aligned}
\ln f(z) & =\ln e^{\int^{z} \frac{1}{t}\left(\frac{1+t}{1-t}\right)^{\lambda} d t}=\int_{0}^{z} \frac{1}{t}\left(\frac{1+t}{1-t}\right)^{\lambda} d t \\
\frac{f^{\prime}(z)}{f(z)} & =\frac{1}{z}\left(\frac{1+z}{1-z}\right)^{\lambda}, \\
h(z) & =z \frac{f^{\prime}(z)}{f(z)}=\left(\frac{1+z}{1-z}\right)^{\lambda}
\end{aligned}
$$

and

$$
h^{\prime}(z)=\lambda\left(\frac{1+z}{1-z}\right)^{\lambda-1} \frac{2}{(1-z)^{2}} .
$$

Therefore, we obtain

$$
h^{\prime}(i)=\lambda\left(\frac{1+i}{1-i}\right)^{\lambda-1} \frac{2}{(1-i)^{2}}
$$

and

$$
\left|h^{\prime}(i)\right|=\lambda=\frac{\beta}{\beta+\alpha} .
$$

Theorem 2.2. Under the same assumptions as in Theorem 2.1, we have

$$
\left|\left(\frac{z f^{\prime}(z)}{f(z)}\right)_{z=b}^{\prime}\right| \geq \frac{4 \beta^{2}}{(\beta+\alpha)\left(2 \beta+(\beta+\alpha)\left|c_{2}\right|\right)} .
$$

The inequality (2.2) is sharp with equality for the function

$$
f(z)=e^{\int_{0}^{z} \frac{1}{t}\left(\frac{1+t}{1-t}\right)^{\lambda} d t}
$$

where $\lambda=\frac{\beta}{\beta+\alpha}$.

Proof. Let $\phi(z)$ be as in the proof of Theorem 2.1. Using the inequality (1.4) for the function $\phi(z)$, we obtain

$$
\frac{2}{1+\left|\phi^{\prime}(0)\right|} \leq\left|\phi^{\prime}(b)\right|=\frac{2}{\lambda}\left|\frac{(h(b))^{\frac{1}{\lambda}-1} h^{\prime}(b)}{\left(1+(h(b))^{\frac{1}{\lambda}}\right)^{2}}\right|=\frac{2}{\lambda} \frac{\left|h^{\prime}(b)\right|}{|1+i|^{2}}=\frac{\left|h^{\prime}(b)\right|}{\lambda} .
$$


Since

$$
\phi^{\prime}(z)=\frac{2}{\lambda} \frac{(h(z))^{\frac{1}{\lambda}-1} h^{\prime}(z)}{\left(1+(h(z))^{\frac{1}{\lambda}}\right)^{2}}
$$

and

$$
\left|\phi^{\prime}(0)\right|=\frac{2}{\lambda}\left|\frac{(h(0))^{\frac{1}{\lambda}-1} h^{\prime}(0)}{\left(1+(h(0))^{\frac{1}{\lambda}}\right)^{2}}\right|=\frac{2}{\lambda} \frac{\left|c_{2}\right|}{4}=\frac{\left|c_{2}\right|}{2 \lambda},
$$

we have

$$
\frac{2}{1+\frac{\left|c_{2}\right|}{2 \lambda}} \leq \frac{\left|h^{\prime}(b)\right|}{\lambda}
$$

and

$$
\left|h^{\prime}(b)\right| \geq \frac{4 \lambda^{2}}{2 \lambda+\left|c_{2}\right|}
$$

So, we obtain the inequality (2.2).

To show that the inequality (2.2) is sharp, take the holomorphic function

$$
f(z)=e^{\int^{z} \frac{1}{t}\left(\frac{1+t}{1-t}\right)^{\lambda} d t}
$$

Then

$$
h(z)=z \frac{f^{\prime}(z)}{f(z)}=\left(\frac{1+z}{1-z}\right)^{\lambda}
$$

and

$$
\left|h^{\prime}(i)\right|=\lambda \text {. }
$$

Since $\left|c_{2}\right|=2 \lambda$ is satisfied with equality. That is;

$$
\frac{4 \lambda^{2}}{2 \lambda+\left|c_{2}\right|}=\frac{4 \lambda^{2}}{2 \lambda+2 \lambda}=\lambda \text {. }
$$

Theorem 2.3. Let $f(z) \in \mathcal{N}(\lambda)$. Assume that, for some $b \in \partial E$, $f$ has angular limit $f(b)$ at $b$ and $\frac{b f^{\prime}(b)}{f(b)}=i^{\lambda}$. Then we have the inequality

$$
\left|\left(\frac{z f^{\prime}(z)}{f(z)}\right)_{z=b}^{\prime}\right| \geq \lambda\left(1+\frac{2\left(2 \lambda-\left|c_{2}\right|\right)^{2}}{4 \lambda^{2}-\left|c_{2}\right|^{2}+\left|4 \lambda c_{3}-c_{2}^{2}(2 \lambda-1)+(1-\lambda) c_{2}\right|}\right),
$$

where $\lambda=\frac{\beta}{\beta+\alpha}$. The inequality (2.3) is sharp with equality for the function

$$
f(z)=e^{\int^{z} \frac{1}{t}\left(\frac{1+t}{1-t}\right)^{\lambda} d t} .
$$

Proof. Let $\phi(z)$ be as in the proof of Theorem 2.1. By the maximum principle for each $z \in E$, we have $|\phi(z)| \leq|z|$. So,

$$
\psi(z)=\frac{\phi(z)}{z}
$$


is a holomorphic function in $E$ and $|\psi(z)|<1$ for $|z|<1$. For any real number $\mu=\frac{1}{\lambda}$ that is not a non-negative integer

$$
k^{\mu}=\sum_{n=0}^{\infty}\left(\begin{array}{l}
\mu \\
n
\end{array}\right)(k-1)^{n}
$$

where $k=\frac{z f^{\prime}(z)}{f(z)}=1+c_{2} z+\left(2 c_{3}-c_{2}^{2}\right) z^{2}+\cdots$.

From equality of $\psi(z)$, we have

$$
\psi(z)=\frac{\phi(z)}{z}=\frac{1}{z} \frac{(h(z))^{\frac{1}{\lambda}}-1}{(h(z))^{\frac{1}{\lambda}}+1}=\frac{1}{z} \frac{(k)^{\mu}-1}{(k)^{\mu}+1} .
$$

Thus, we take

$$
|\psi(0)|=\frac{\left|c_{2}\right|}{2 \lambda} \leq 1
$$

and

$$
\left|\psi^{\prime}(0)\right|=\frac{\left|4 \lambda c_{3}-c_{2}^{2}(2 \lambda-1)+(1-\lambda) c_{2}\right|}{4 \lambda^{2}} .
$$

Moreover, it can be seen that

$$
\frac{b \phi^{\prime}(b)}{\phi(b)}=\left|\phi^{\prime}(b)\right| \geq\left|\left(b^{p}\right)^{\prime}\right|=\frac{b\left(b^{p}\right)^{\prime}}{b^{p}} .
$$

The function

$$
\Phi(z)=\frac{\psi(z)-\psi(0)}{1-\overline{\psi(0)} \psi(z)}
$$

is a holomorphic in the unit disc $E,|\Phi(z)|<1$ for $|z|<1, \Phi(0)=0$ and $|\Phi(b)|=1$ for $b \in \partial E$.

From (1.4), we obtain

$$
\begin{aligned}
\frac{2}{1+\left|\Phi^{\prime}(0)\right|} & \leq\left|\Phi^{\prime}(b)\right|=\frac{1-|\psi(0)|^{2}}{|1-\overline{\psi(0)} \psi(b)|^{2}}\left|\psi^{\prime}(b)\right| \leq \frac{1+|\psi(0)|}{1-|\psi(0)|}\left|\psi^{\prime}(b)\right| \\
& =\frac{1+|\psi(0)|}{1-|\psi(0)|}\left\{\left|\phi^{\prime}(b)\right|-1\right\} .
\end{aligned}
$$

Since

$$
\begin{aligned}
\Phi^{\prime}(z) & =\frac{1-|\psi(0)|^{2}}{(1-\overline{\psi(0)} \psi(z))^{2}} \psi^{\prime}(z) \\
\left|\Phi^{\prime}(0)\right| & =\frac{\left|\psi^{\prime}(0)\right|}{1-|\psi(0)|^{2}}=\frac{\frac{\left|4 \lambda c_{3}-c_{2}^{2}(2 \lambda-1)+(1-\lambda) c_{2}\right|}{4 \lambda^{2}}}{1-\left(\frac{\left|c_{2}\right|}{2 \lambda}\right)^{2}}=\frac{\left|4 \lambda c_{3}-c_{2}^{2}(2 \lambda-1)+(1-\lambda) c_{2}\right|}{4 \lambda^{2}-\left|c_{2}\right|^{2}},
\end{aligned}
$$


we take

$$
\begin{aligned}
\frac{2}{1+\frac{\left|4 \lambda c_{3}-c_{2}^{2}(2 \lambda-1)+(1-\lambda) c_{2}\right|}{4 \lambda^{2}-\left|c_{2}\right|^{2}}} & \leq \frac{1+\frac{\left|c_{2}\right|}{2 \lambda}}{1-\frac{\left|c_{2}\right|}{2 \lambda}}\left\{\frac{\left|h^{\prime}(b)\right|}{\lambda}-1\right\} \\
& =\frac{2 \lambda+\left|c_{2}\right|}{2 \lambda-\left|c_{2}\right|}\left\{\frac{\left|h^{\prime}(b)\right|}{\lambda}-1\right\} .
\end{aligned}
$$

Therefore, we obtain

$$
1+\frac{2\left(4 \lambda^{2}-\left|c_{2}\right|^{2}\right)}{4 \lambda^{2}-\left|c_{2}\right|^{2}+\left|4 \lambda c_{3}-c_{2}^{2}(2 \lambda-1)+(1-\lambda) c_{2}\right|} \frac{2 \lambda-\left|c_{2}\right|}{2 \lambda+\left|c_{2}\right|} \leq \frac{\left|h^{\prime}(b)\right|}{\lambda}
$$

and

$$
\left|h^{\prime}(b)\right| \geq \lambda\left(1+\frac{2\left(2 \lambda-\left|c_{2}\right|\right)^{2}}{4 \lambda^{2}-\left|c_{2}\right|^{2}+\left|4 \lambda c_{3}-c_{2}^{2}(2 \lambda-1)+(1-\lambda) c_{2}\right|}\right) .
$$

So, we obtain the inequality (2.3).

To show that the inequality (2.3) is sharp, take the holomorphic function

$$
f(z)=e^{\int^{0} \frac{1}{t}\left(\frac{1+t}{1-t}\right)^{\lambda} d t}
$$

Then

$$
h(z)=z \frac{f^{\prime}(z)}{f(z)}=\left(\frac{1+z}{1-z}\right)^{\lambda}
$$

and

$$
\left|h^{\prime}(i)\right|=\lambda \text {. }
$$

Since $\left|c_{2}\right|=2 \lambda,(2.3)$ is satisfied with equality.

If $\left(\frac{z f^{\prime}(z)}{f(z)}\right)^{\frac{1}{\lambda}}-1$ has no zeros different from $z=0$ in Theorem 2.3 , the inequality (2.3) can be further strengthened. This is given by the following theorem.

Theorem 2.4. Let $f(z) \in \mathcal{N}(\lambda)$ and $\left(\frac{z f^{\prime}(z)}{f(z)}\right)^{\frac{1}{\lambda}}-1$ has no zeros in $E$ except $z=0$ and $c_{2}>0$. Assume that, for some $b \in \partial E, f$ has angular limit $f(b)$ at $b$ and $\frac{b f^{\prime}(b)}{f(b)}=i^{\lambda}$. Then we have the inequality

$$
\left|\left(\frac{z f^{\prime}(z)}{f(z)}\right)_{z=b}^{\prime}\right| \geq \lambda\left(1-\frac{2 \lambda\left|c_{2}\right| \ln ^{2}\left(\frac{\left|c_{2}\right|}{2 \lambda}\right)}{2 \lambda\left|c_{2}\right| \ln \left(\frac{\left|c_{2}\right|}{2 \lambda}\right)-\left|4 \lambda c_{3}-c_{2}^{2}(2 \lambda-1)+(1-\lambda) c_{2}\right|}\right),
$$

where $\lambda=\frac{\beta}{\beta+\alpha}$. In addition, the equality in (2.5) occurs for the function

$$
f(z)=e^{\int_{0}^{z} \frac{1}{t}\left(\frac{1+t}{1-t}\right)^{\lambda} d t}
$$

where $\lambda=\frac{\beta}{\beta+\alpha}$. 
Proof. Let $c_{2}>0$ in the expression of the function $f(z)$. Having in mind the inequality (2.4) and the function $\left(\frac{z f^{\prime}(z)}{f(z)}\right)^{\frac{1}{\lambda}}-1$ has no zeros in $E$ except $E-\{0\}$, we denote by $\ln \psi(z)$ the holomorphic branch of the logarithm normed by the condition

$$
\ln \psi(0)=\ln \left(\frac{\left|c_{2}\right|}{2 \lambda}\right)<0
$$

The auxiliary function

$$
\Delta(z)=\frac{\ln \psi(z)-\ln \psi(0)}{\ln \psi(z)+\ln \psi(0)}
$$

is a holomorphic in the unit disc $E,|\Delta(z)|<1, \Delta(0)=0$ and $|\Delta(b)|=1$ for $b \in \partial E$.

From (1.4), we obtain

$$
\begin{aligned}
\frac{2}{1+\left|\Delta^{\prime}(0)\right|} & \leq\left|\Delta^{\prime}(b)\right|=\frac{|2 \ln \psi(0)|}{|\ln \psi(b)+\ln \psi(0)|^{2}}\left|\frac{\psi^{\prime}(b)}{\psi(b)}\right| \\
& =\frac{-2 \ln \psi(0)}{\ln ^{2} \psi(0)+\arg ^{2} \psi(b)}\left\{\left|\phi^{\prime}(b)\right|-1\right\} .
\end{aligned}
$$

Since

$$
\begin{aligned}
\left|\Delta^{\prime}(0)\right| & =\frac{-1}{\ln \left(\frac{\left|c_{2}\right|}{2 \lambda}\right)} \frac{\frac{\left|4 \lambda c_{3}-c_{2}^{2}(2 \lambda-1)+(1-\lambda) c_{2}\right|}{4 \lambda^{2}}}{\frac{\left|c_{2}\right|}{2 \lambda}} \\
& =\frac{-1}{\ln \left(\frac{\left|c_{2}\right|}{2 \lambda}\right)} \frac{\left|4 \lambda c_{3}-c_{2}^{2}(2 \lambda-1)+(1-\lambda) c_{2}\right|}{2 \lambda\left|c_{2}\right|}
\end{aligned}
$$

and replacing $\arg ^{2} \psi(b)$ by zero, then we have

$$
\frac{1}{1-\frac{\left|4 \lambda c_{3}-c_{2}^{2}(2 \lambda-1)+(1-\lambda) c_{2}\right|}{2 \lambda\left|c_{2}\right| \ln \left(\frac{\left|c_{2}\right|}{2 \lambda}\right)}} \leq \frac{-1}{\ln \left(\frac{\left|c_{2}\right|}{2 \lambda}\right)}\left\{\frac{\left|h^{\prime}(b)\right|}{\lambda}-1\right\}
$$

and

$$
1-\frac{2 \lambda\left|c_{2}\right| \ln ^{2}\left(\frac{\left|c_{2}\right|}{2 \lambda}\right)}{2 \lambda\left|c_{2}\right| \ln \left(\frac{\left|c_{2}\right|}{2 \lambda}\right)-\left|4 \lambda c_{3}-c_{2}^{2}(2 \lambda-1)+(1-\lambda) c_{2}\right|} \leq \frac{\left|h^{\prime}(b)\right|}{\lambda} .
$$

Thus, we obtain the inequality (2.5) with an obvious equality case.

The following inequality (2.6) is weaker, but is simpler than (2.5) and does not contain the coeffient $c_{3}$.

Theorem 2.5. Under the hypotheses of Theorem 2.4, we have the inequality

$$
\left|\left(\frac{z f^{\prime}(z)}{f(z)}\right)_{z=b}^{\prime}\right| \geq \frac{\beta}{\beta+\alpha}\left[1-\ln \left((\beta+\alpha) \frac{\left|c_{2}\right|}{2 \beta}\right)\right] .
$$


Moreover, the result is sharp and the extremal function is

$$
f(z)=e^{\int_{0}^{z} \frac{1}{t}\left(\frac{1+t}{1-t}\right)^{\lambda} d t}
$$

where $\lambda=\frac{\beta}{\beta+\alpha}$.

Proof. Let $c_{2}>0$. Using the inequality (1.5) for the function $\Phi(z)$, we obtain

$$
1 \leq\left|\Delta^{\prime}(b)\right|=\frac{|2 \ln \psi(0)|}{|\ln \psi(b)+\ln \psi(0)|^{2}}\left|\frac{\psi^{\prime}(b)}{\psi(b)}\right|=\frac{-2 \ln \psi(0)}{\ln ^{2} \psi(0)+\arg ^{2} \psi(b)}\left\{\left|\phi^{\prime}(b)\right|-1\right\} .
$$

Replacing $\arg ^{2} \varphi(b)$ by zero, then we have

$$
1 \leq \frac{-1}{\ln \left(\frac{\left|c_{2}\right|}{2 \lambda}\right)}\left\{\frac{\left|h^{\prime}(b)\right|}{\lambda}-1\right\}
$$

and

$$
\left|h^{\prime}(b)\right| \geq \lambda\left[1-\ln \left(\frac{\left|c_{2}\right|}{2 \lambda}\right)\right]
$$

Thus, we obtain the inequality (2.6) with an obvious equality case.

\section{REFERENCES}

[1] T. A. Azeroğlu and B. Örnek, A refined schwarz inequality on the boundary, Complex Var. Elliptic Equ. 58 (2013), 571-577.

[2] H. P. Boas, Julius and Julia: mastering the art of the Schwarz lemma, Amer. Math. Monthly 117 (2010), 770-785.

[3] D. M. Burns and S. G. Krantz, Rigidity of holomorphic mappings and a new Schwarz lemma at the boundary, J. Amer. Math. Soc. 7 (1994), 661-676.

[4] D. Chelst, A generalized Schwarz lemma at the boundary, Proc. Amer. Math. Soc. 129 (2001), $3275-3278$.

[5] V. Dubinin, The Sschwarz inequality on the boundary for functions regular in the disk, J. Math. Sci. 122 (2004), 3623-3629.

[6] V. Dubinin, Bounded holomorphic functions covering no concentric circles, J. Math. Sci. 207(6) (2015), 825-831.

[7] M. Elin, F. Jacobzon, M. Levenshtein and D. Shoikhet, The Schwarz lemma: rigidity and dynamics, in: Harmonic and Complex Analysis and its Applications, Springer, Switzerland, Basel, 2014, 135-230.

[8] G. M. Goluzin, Geometric Theory of Functions of a Complex Variable, American Mathematical Society, Providence, Rhode Island, 1969.

[9] M. Jeong, The Schwarz lemma and its application at a boundary point, Pure Appl. Math. 21 (2014), 219-227.

[10] M.-J. Jeong, The Schwarz lemma and boundary fixed points, Pure Appl. Math. 18 (2011), 275-284.

[11] M. Mateljević, Note on rigidity of holomorphic mappings $\&$ Schwarz and Jack lemma, Filomat, (to appear).

[12] M. Mateljević, Ahlfors-Schwarz lemma and curvature, Kragujevac J. Math. 25 (2003), 155-164.

[13] M. Mateljevic, Distortion of harmonic functions and harmonic quasiconformal quasi-isometry, Rev. Roumaine Math. Pures Appl. 51 (2006), 711-722. 
[14] M. Mateljević, The lower bound for the modulus of the derivatives and jacobian of harmonic injective mappings, Filomat 29 (2015), 221-244.

[15] B. Ornek, Estimates for holomorphic functions concerned with Jack's lemma, Publ. Inst. Math. (Beograd) (N.S.) 104(118) (2018), 231-240.

[16] B. N. Ornek, Sharpened forms of the Schwarz lemma on the boundary, Bull. Korean Math. Soc. 50 (2013), 2053-2059.

[17] R. Osserman, A sharp Schwarz inequality on the boundary, Proc. Amer. Math. Soc. 128 (2000), 3513-3517.

[18] C. Pommerenke, Boundary Behaviour of Conformal Maps, Grundlehren der mathematischen Wissenschaften 299, Springer-Verlag, Berlin, Heidelberg, 1992.

[19] X. Tang and T. Liu, The Schwarz lemma at the boundary of the egg domain $B_{p_{1}, p_{2}}$ in $\mathbb{C}^{n}$, Canad. Math. Bull. 58 (2015), 381-392.

[20] X. Tang, T. Liu and J. Lu, Schwarz lemma at the boundary of the unit polydisk in $\mathbb{C}^{n}$, Sci. China Math. 58 (2015), 1639-1652.

[21] H. Unkelbach, Über die randverzerrung bei konformer abbildung, Math. Z. 43 (1938), 739-742.

${ }^{1}$ Department of Computer Engineering

AmASYA UNIVERSITY,

MERKEZ-AMASYA 05100, TURKEY

Email address: nafiornek@gmail.com, nafi.ornek@amasya.edu.tr 